\title{
Photo-decomposable Organic Nanoparticles for Combined Tumor Optical Imaging and Multiple Phototherapies
}

Wenjun Miao, ${ }^{1,2}$, Hyungjun Kim¹, Vipul Gujrati ${ }^{1}$, Jin Yong Kim ${ }^{3}$, Hyeongsu Jon¹, Yonghyun Lee ${ }^{1}$, Minsuk Choi $^{1}$, Jinjoo Kim ${ }^{1}$, Soyoung Lee ${ }^{1}$, Dong Yun Lee ${ }^{3}$, Sukmo Kang ${ }^{1}$, Sangyong Jon ${ }^{1,3} \bowtie$

1. KAIST Institute for the BioCentury, Department of Biological Sciences, Korea Advanced Institute of Science and Technology, Daejon 305-701, Republic of Korea.

2. School of Pharmaceutical Science, Nanjing Tech University, Nanjing, 211816, China.

3. Graduate School of Medical Science and Engineering, Korea Advanced Institute of Science and Technology, Daejon 305-701, Republic of Korea.

$\bowtie$ Corresponding author: Sangyong Jon. (email: syjon@kaist.ac.kr).

(C) Ivyspring International Publisher. Reproduction is permitted for personal, noncommercial use, provided that the article is in whole, unmodified, and properly cited. See http://ivyspring.com/terms for terms and conditions.

Received: 2016.04.12; Accepted: 2016.06.07; Published: 2016.10.01

\begin{abstract}
Combination of photodynamic therapy (PDT) with photothermal therapy (PTT) has achieved significantly improved therapeutic efficacy compared to a single phototherapy modality. However, most nanomaterials used for combined PDT/PTT are made of non-biodegradable materials (e.g., gold nanorods, carbon nanotubes, and graphenes) and may remain intact in the body for long time, raising concerns over their potential long-term toxicity. Here we report a new combined PDT/PTT nanomedicine, designated SP3NPs, that exhibit photo-decomposable, photodynamic and photothermal properties. SP ${ }^{3} \mathrm{NPs}$ were prepared by self-assembly of PEGylated cypate, comprising FDA-approved PEG and an ICG derivative. We confirmed the ability of SP'NPs to generate both singlet oxygen for a photodynamic effect and heat for photothermal therapy in response to NIR laser irradiation in vitro. Also, the unique ability of SP ${ }^{3} \mathrm{NPs}$ to undergo irreversible decomposition upon NIR laser irradiation was demonstrated. Further our experimental results demonstrated that $S P^{3} N P s$ strongly accumulated in tumor tissue owing to their highly PEGylated surface and relatively small size $(\sim 60 \mathrm{~nm})$, offering subsequent imaging-guided combined PDT/PTT treatment that resulted in tumor eradication and prolonged survival of mice. Taken together, our SP3NPs described here may represent a novel and facile approach for next-generation theranostics with great promise for translation into clinical practice in the future.
\end{abstract}

Key words: nanomedicine $\cdot$ indocyanine green $\cdot$ photodynamic therapy $\cdot$ photothermal therapy $\cdot$ theranostics.

\section{Introduction}

Nanotechnology-assisted phototherapy - the use of light in conjunction with light-responsive nanoparticles - has been shown to be a promising strategy for cancer treatment because nanoparticles lead to preferential accumulation in tumors through the enhanced permeability and retention (EPR) effect and the subsequent phototherapy enables precise destruction of tumors in a spatiotemporal manner.[1-6] However, a single phototherapy modality alone, such as photodynamic therapy (PDT) or photothermal therapy (PTT), has shown limited therapeutic efficacy in vivo. PDT depletes oxygen in tumor tissues and destroys tumor-associated blood vessels, resulting in a significant reduction in blood flow to the tumor and consequent severe local hypoxia, which is responsible for poor responses to subsequent radio- and chemotherapy.[7-10] PTT, which relies on the conversion of near-infrared (NIR) light to heat using photo-responsive nanomaterials, usually leads to incomplete tumor destruction owing 
to uneven heat transfer and distribution within the tumor, resulting in tumor recurrence initiated by surviving cancer cells in the residual tumor mass.[6, 11-14] Very recent attempts to combine PDT with PTT so as to overcome the limitations associated with a single phototherapy modality have achieved significantly improved therapeutic efficacy.[15-23] The appreciable local hyperthermia created by PTT increases intratumoral blood flow and oxygenation, which is favorable for PDT. Conversely, reactive oxygen species (ROS) generated by PDT sensitize cancer cells to the hyperthermia generated by PTT. However, previously reported systems for combined PDT/PTT, constructed by loading or conjugating known photosensitizers (PS) onto NIR-absorbing nanoparticles, still have some drawbacks. First, because of the mismatch in absorption wavelengths of PS and NIR-responsive nanoparticles, two lasers are needed to excite them separately,[15, 19-20, 24] complicating the treatment process. Second, PS-loaded NIR nanoparticles show appreciably reduced production of singlet oxygen $\left({ }^{1} \mathrm{O}_{2}\right)$ species as a result of Förster resonance energy transfer between PS and NPs within close proximity,[21, 25-26] leading to compromised PDT efficacy. Third, most NIR nanoparticles used for PTT are made of non-biodegradable materials (e.g., carbon, gold and other noble metal-based nanomaterials) and may remain intact in the body for long time,[16-18, 26] raising concerns over their potential long-term toxicity. Additionally, complexities in fabrication or manufacturing processes for previously developed combined PDT and PTT systems may further hamper their future translation to the clinic. Thus, there is a pressing need to develop a new combined PDT and PTT nanomedicine that (1) enables effective cancer therapy and imaging using a single laser, (2) is degradable and excretable, and (3) can be easily scaled up.

Indocyanine green (ICG), a clinically used NIR cyanine dye, may be a good starting material for the design of nanomedicines that satisfy these criteria because it is known to exhibit both PDT and PTT effects, [27-29] and can also undergo decomposition into smaller fragments upon NIR irradiation.[30] Despite such favorable properties of ICG for use in multiple phototherapies, however, most previous studies have been limited to use of ICG for optical imaging and/or single phototherapy (i.e., PDT or PTT) by physical entrapment of the dye into various types of nanoparticles such as micelles and liposomes.[31-39] Although such ICG-loaded nanoparticles have shown marked progress in terms of enhanced photo-stability and circulation time compared to the pristine dye, there has been few nanoparticles that fully take advantages of ICG's photonic properties, including photo-decomposability, optical imaging, PDT, and PTT. A plausible explanation on the limited use of ICG is that it is challenging to covalently conjugate ICG with nanomaterials because it lacks modifiable chemical functionalities. Cypate, an analog of ICG, has similar photo-properties as ICG, but unlike ICG it possesses functional groups available for modification (two carboxylic acids) and a hydrophobic skeleton for assembly.[40] With these considerations in mind, we, for the first time, designed cypate-based nanoparticles for use in combined PDT/PTT therapy. To form cypate-based nanostructures, we introduced a hydrophilic, biocompatible polyethylene glycol (PEG) to cypate, generating PEGylated cypate. We expected that the amphiphilic, PEGylated cypate would spontaneously self-assemble into small nanoparticles, designated $\mathrm{SP}^{3} \mathrm{NPs}$, that exhibit photo-decomposable, photodynamic and photothermal properties (Figure 1A). It was anticipated that SP ${ }^{3} \mathrm{NPs}$ would effectively accumulate in tumor sites via the EPR effect, emit NIR-range fluorescence for tumor optical imaging, and upon irradiation using a single NIR laser, would generate reactive singlet oxygen together with heat, thus constituting an imaging-guided, multiple phototherapy modality (combined PDT/PTT) for treating cancer.

\section{Materials and Methods}

\section{Materials}

Cypate was synthesized according to previous reports.[34] Methoxypolyethylene glycol amine (mPEG $\left.{ }_{2000}-\mathrm{NH}_{2}\right), \quad \mathrm{N}, \mathrm{N}^{\prime}$-dicyclohexylcarbodiimide (DCC), N-hydroxysuccinimide (NHS), 3-(4,5-dimethylthiazol-2-yl)-2,5- diphenyltetrazolium bromide (MTT) and 4',6-diamidino-2-phenylindole dihydrochloride (DAPI) were purchased from Sigma-Aldrich (St, Louis, MO). Singlet Oxygen Sensor Green (SOSG) and ROS-detection dye, 2',7'-Dichlorodihydrofluorescein diacetate $\left(\mathrm{H}_{2} \mathrm{DCFDA}\right)$ were obtained from Thermo Fisher Scientific Inc. (Grand Island, NY). Mounting solution for confocal microscopy was purchased from Dako (Glostrup, Denmark). ApoBrdU DNA fragmentation assay kit for TUNEL assay was supplied by BioVision Inc. (Milpitas, CA). All other chemicals were of reagent grade and were used as received.

Murine melanoma B16F10 cells were supplied by American Type Culture Collection (ATCC, Rockville, MD). Cell culture media DMEM and fetal bovine serum (FBS) were purchased from Gibco-BRL Life Technologies (Carlsbad, CA). Male C57BL/6 mice aged 6-8 wk were obtained from Orient Bio. Lab. 
Animal Inc. (Seungnam, Kyonggi-do, South Korea). All animal experiments were conducted in accordance with the Guidelines of the Korea Advanced Institute of Science and Technology Institutional Animal Care and Use Committee (KAIST-IACUC).

\section{Synthesis of PEGylated cypate}

PEGylated cypate was synthesized by conjugating cypate with $\mathrm{mPEG}_{2000}-\mathrm{NH}_{2}$ via an amide bond. In brief, DCC $(90 \mu \mathrm{mol})$ and NHS $(90 \mu \mathrm{mol})$ were added to cypate $(60 \mu \mathrm{mol})$ in dichloromethane $(10 \mathrm{ml})$. After stirring for $30 \mathrm{~min}$, insoluble byproducts were removed by filtration. $\mathrm{mPEG}_{2000}-\mathrm{NH}_{2}(20 \mu \mathrm{mol})$ was added to the activated cypate, and the mixture was reacted for $24 \mathrm{~h}$ at room temperature. The crude product was purified by silica gel column chromatography using chloroform/methanol (75:25, $\mathrm{v} / \mathrm{v})$. The obtained PEGylated cypate was characterized by ${ }^{1} \mathrm{H}$ NMR using a Bruker AVANCE-500MHz FT-NMR spectrometer (Bruker, Billerica, MA). The molecular weight of PEGylated cypate was measured by matrix-assisted laser desorption/ionization-time of flight spectrometry (MALDI-TOF) using an Autoflex III MALD-TOF system (Bruker).

\section{Preparation and characterization of SP ${ }^{3}$ NPs}

PEGylated cypate in methanol was dried under rotary evaporation, and the resulting thin film was hydrated with $1 \mathrm{ml}$ of PBS (pH 7.4) and vortexed. After sonication for $10 \mathrm{~min}$, large aggregates were removed by filtration through $0.2-\mu \mathrm{m}$ polycarbonate membrane filters (Millipore Corp., Billerica, MA), and $\mathrm{SP}^{3} \mathrm{NPs}$ were obtained and stored at $4{ }^{\circ} \mathrm{C}$ until use. The size and morphology of $\mathrm{SP}^{3} \mathrm{NPs}$ were examined by TEM using a JEM1010 system (Jeol Ltd, Tokyo, Japan). The hydrodynamic diameters and zeta potentials of $\mathrm{SP}^{3} \mathrm{NPs}$ were measured using a Nanosizer ZS90 instrument (Malvern Instruments Ltd, Malvern, UK). The colloidal stability of $\mathrm{SP}^{3} \mathrm{NPs}$ in PBS and 50\% FBS was tested by monitoring changes in size over $1 \mathrm{wk}$. The UV-Vis spectra of SP ${ }^{3} \mathrm{NPs}$ were recorded using a UV-Vis spectrophotometer (NEOSYS2000; Scinco, Twin Lakes, WI).

\section{NIR irradiation-triggered decomposition and photo-properties of SP ${ }^{3}$ NPs}

SP ${ }^{3} N P s\left(100 \mu \mathrm{g} \mathrm{ml}^{-1}\right)$ in PBS were irradiated for 10 min using a BWF2 continuous-wave NIR laser (808 $\mathrm{nm}, 0.8 \mathrm{~W} \mathrm{~cm}^{-2}$; B\&W Tek Inc., Newark, DE). Changes in absorbance spectra and morphology of SPNPs during irradiation were monitored using a UV-Vis spectrophotometer and TEM, respectively. The appearance of $\mathrm{SP}^{3} \mathrm{NPs}$ during NIR irradiation was recorded using a digital camera.
Fluorescence images of free cypate and $\mathrm{SP}^{3} \mathrm{NPs}$ in PBS at the same concentrations were recorded with a Xenogen IVIS Lumina imaging system (Perkin Elmer Inc., Waltham, MA) with a built-in ICG filter set using an exposure time of $5 \mathrm{~s}$. The fluorescence intensity in each region of interest (ROI) was semi-quantitated by Xenogen Living Image ${ }^{\circledR}$, and plotted against the concentration of free cypate and SPNPs. Photodynamic effects were tested by irradiating free cypate and SP3 NPs $\left(25 \mu \mathrm{g} \mathrm{ml}^{-1}\right)$ in the presence and absence of sodium azide $(100 \mu \mathrm{M})$ for 5 $\min \left(808 \mathrm{~nm}, 0.8 \mathrm{~W} \mathrm{~cm}^{-2}\right)$, and the generated singlet oxygen was detected with SOSG following the manufacturer's instructions. PBS served as a negative control. Photothermal effects were assessed by irradiation of different concentrations of $\mathrm{SP}^{3} \mathrm{NPs}$ with an 808-nm NIR laser $\left(0.8 \quad \mathrm{~W} \quad \mathrm{~cm}^{-2}, \quad 5 \mathrm{~min}\right)$. Temperatures were quantified using an IR thermal imaging system (FLIR T420; FLIR Systems Inc., Danderyd, Sweden). The photothermal heating curve of PBS was measured as a negative control.

\section{In vitro cellular-uptake test}

Cellular uptake of $\mathrm{SP}^{3} \mathrm{NPs}$ was determined by confocal microscopy and flow cytometry using the intrinsic fluorescence of cypate. B16F10 cells were cultured in DMEM supplemented with 10\% FBS, 100 units $\mathrm{ml}^{-1}$ penicillin, and $100 \mu \mathrm{g} \mathrm{ml}-1$ streptomycin. B16F10 cells were seeded onto cover glasses at a density of $1 \times 10^{5}$ cells per well in 24 -well plates. After reaching $70 \%$ confluence, cells were treated with free cypate or $\mathrm{SP}^{3} \mathrm{NPs}$ at an equivalent dose of cypate. After incubation for $1 \mathrm{~h}$, cells were washed with cold PBS, fixed with $4 \%$ paraformaldehyde for $15 \mathrm{~min}$, and stained with DAPI. Cellular fluorescence was observed using a confocal laser-scanning microscope (LSM 5 Exciter; Carl Zeiss, Inc., Jena, Germany). Flow cytometry measurements were conducted by first harvesting cells and washing them three times with cold PBS containing 2\% FBS. Cells were then analyzed using a BD FACSCalibur flow cytometer using Cell Quest Pro software (BD Biosciences, San Jose, CA).

\section{Intracellular ROS and heat generation upon irradiation}

B16F10 cells were seeded onto 24-well plates at a density of $1 \times 10^{5}$ cells per well. The following day, cells were treated with free cypate or SP3NPs at a concentration of $5 \mu \mathrm{g} \mathrm{ml}^{-1}$ (equivalent dose of cypate) for $1 \mathrm{~h}$. After washing with cold PBS, cells were irradiated with a NIR laser $\left(808 \mathrm{~nm}, 0.8 \mathrm{~W} \mathrm{~cm}^{-2}\right)$ for 3 min and then incubated with fresh media containing $\mathrm{H}_{2}$ DCFDA $(10 \mu \mathrm{M})$ for $1 \mathrm{~h}$. DCF (green) was imaged using a fluorescence microscopy (Leica, DM IL, Germany). The types of generated ROS were 
identified by adding different ROS scavengers, including glutathione, sodium azide (singlet oxygen scavenger), D-mannitol (hydroxyl radical scavenger) or sodium pyruvate (hydrogen peroxide scavenger), to the media during NIR irradiation. Subsequent procedures were as indicated above. Intracellular heat generation was evaluated by first treating B16F10 cells with free cypate or SP ${ }^{3} \mathrm{NPs}\left(20 \mu \mathrm{g} \mathrm{ml}^{-1}\right.$, equivalent to cypate) for $1 \mathrm{~h}$. After washing with cold PBS, cells were exposed to a NIR laser for $3 \mathrm{~min}(808 \mathrm{~nm}, 0.8 \mathrm{~W}$ $\mathrm{cm}^{-2}$ ), and thermal images were recorded using an IR thermal camera, as described above.

\section{Laser-triggered phototoxicity of SP ${ }^{3}$ NPs toward tumor cells}

The in vitro antitumor efficacy of free cypate and $\mathrm{SP}^{3} \mathrm{NPs}$ upon NIR irradiation was tested using MTT assays, as previously described.[41] Briefly, B16F10 cells were seeded onto 96-well plates at a density of 1 $\times 10^{4}$ cells per well. The next day, cells were treated with serial dilutions of free cypate and SP ${ }^{3} \mathrm{NPs}$ for $1 \mathrm{~h}$. After washing with cold PBS, cells were irradiated with an $808 \mathrm{~nm}$ NIR laser for $3 \mathrm{~min}$. Cells were incubated for $24 \mathrm{~h}$, and cell viability was quantified by MTT assay. The cytoprotective effect of different ROS scavengers was tested by treating cells with $\mathrm{SP}^{3} \mathrm{NPs}$ $\left(100 \mu \mathrm{g} \mathrm{ml}^{-1}\right)$ for $1 \mathrm{~h}$ and further incubating with media containing ROS scavengers (sodium pyruvate, glutathione, sodium azide or D-mannitol, $100 \mu \mathrm{M}$ ) for $30 \mathrm{~min}$ before MTT assay. The cytotoxicity of NIR-degraded fragments of SP NPs was tested by completely decomposing SP3NPs with a NIR laser, and treating B16F10 cells for $24 \mathrm{~h}$ with the resulted colorless products. Cell viability was quantitated by MTT assay and expressed relative to that of an untreated control group.

\section{In vivo tumor-detection ability of SP ${ }^{3} \mathrm{NPs}$}

Depilated C57BL/6 mice were subcutaneously inoculated in the dorsal right side with $1 \times 10^{6}$ B16F10 cells, and tumors were allowed to become established over time. When the tumor volume reached approximately $100 \mathrm{~mm}^{3}$, free cypate or SP3NPs at an equivalent cypate dose of $1 \mathrm{mg} \mathrm{kg}^{-1}$ was injected through the tail vein. At predetermined time points post-administration, the in vivo distribution of fluorescent free cypate and $\mathrm{SP}^{3} \mathrm{NPs}$ within the mice was assessed using a Xenogen IVIS Lumina imaging system (Perkin Elmer Inc.) with a built-in ICG filter set and an exposure time of $5 \mathrm{~s}$. The ex vivo biodistribution patterns of cypate and $\mathrm{SP}^{3} \mathrm{NPs}$ were evaluated by a fluorescence imaging system as well. In some experiments, tumors were excised and cryosectioned, and the intratumoral distribution of free cypate or $\mathrm{SP}^{3} \mathrm{NPs}$ was assessed using confocal microscopy, as described above.

\section{In vivo tumor ablation by SP ${ }^{3}$ NPs upon NIR irradiation}

B16F10 tumor-bearing mice were randomized into six groups based on body weight and tumor volume ( $\mathrm{n}=6-7$ mice/group): (1) PBS, (2) PBS + NIR irradiation, (3) free cypate, (4) free cypate + NIR irradiation, (5) SP3${ }^{3} \mathrm{NPs}$, (6) SP ${ }^{3} \mathrm{NPs}+$ NIR irradiation. Mice were administered a single intravenous dose of PBS, free cypate or SP NPs at the same cypate dose (5 $\mathrm{mg} \mathrm{kg}^{-1}$ ). At $4 \mathrm{~h}$ post-administration, mice were anesthetized and positioned in a holder. The tumor sites were irradiated with an 808-nm continuous-wave NIR laser $\left(0.4 \mathrm{~W} \mathrm{~cm}^{-2}, 10 \mathrm{~min}\right)$. Light-induced temperature changes in the tumor sites were recorded using a real-time IR thermal imaging system (FLIR T420). After treatment, tumor volumes, body weight, tumor appearance, and survival were monitored. Tumor volume was determined by measuring in two dimensions using an electronic caliper, and calculated according to the equation a $\times$ $\mathrm{b}^{2} \times 0.5$, where $\mathrm{a}$ is the largest and $\mathrm{b}$ is the smallest dimension. When the tumor volume reached 2000 $\mathrm{mm}^{3}$, the mice were euthanized in accord with KAIST-IACUC. In some experiments, tumor tissues were extracted $12 \mathrm{~h}$ after irradiation, fixed in $4 \%$ paraformaldehyde, embedded in paraffin, and sectioned at a thickness of $10 \mu \mathrm{m}$. Tumor tissue slides were stained with H\&E and observed using optical microscopy. Apoptotic cells in tissue sections were visualized by TUNEL assay using ApoBrdU DNA fragmentation assay kit according to the manufacturer's instructions.

\section{Statistics}

Analysis of variance (ANOVA) was used to analyze experimental data in conjunction with a Student-Newman-Keuls test for post hoc pairwise comparisons. All statistical analyses were done using SigmaStat software (version 3.5; Systat Software, Richmond, CA); a p-value $<0.05$ was considered significant.

\section{Results and Discussion}

\section{Synthesis and characterization of SP ${ }^{3}$ NPs.}

PEGylated cypate was synthesized in a one-step process by reacting the carbodiimide-activated form of one of two carboxylic acids in cypate with mPEG ${ }_{2000}-\mathrm{NH}_{2}$ (Figure 1A), and was characterized by ${ }^{1} \mathrm{H}$ NMR and mass spectrometry (Figure S1). When placed in an aqueous solution, PEGylated cypate spontaneously self-assembled into micellar-type nanoparticles ( $\mathrm{SP}^{3} \mathrm{NPs}$ ) in which the hydrophobic cypate constitutes the core of the nanoparticle and the 
hydrophilic PEG corona faces toward water, as depicted in Figure 1A. Dynamic light scattering (DLS) measurements of $\mathrm{SP}^{3} \mathrm{NPs}$ showed a narrow size distribution with an average hydrodynamic diameter of $60 \pm 8 \mathrm{~nm}$ (Figure 1B). High-resolution in situ transmission electron microscopy (HRTEM) imaging revealed that, in a dry state, $\mathrm{SP}^{3} \mathrm{NPs}$ had a well-defined spherical nanostructure with a diameter of $\sim 40 \mathrm{~nm}$ (Figure 1C). SP ${ }^{3} \mathrm{NPs}$ also showed good colloidal stability without significant change in size over 1 wk in both PBS and 50\% FBS (Figure 1E). The zeta potential value of SP NPs in PBS at $\mathrm{pH} 7.4$ was $0.06 \pm 0.22 \mathrm{mV}$, suggesting that these particles are nearly neutrally charged. In addition, SP ${ }^{3} \mathrm{NPs}$ in PBS exhibited intense absorption in the NIR region, with a characteristic peak at $790 \mathrm{~nm}$ corresponding to free cypate (Figure 1D). Figure 1F shows NIR fluorescence images of free cypate and SP ${ }^{3} \mathrm{NPs}$ excited at 710-760 $\mathrm{nm}$. And their fluorescence intensities were semi-quantitated and shown in Figure 1G. SP NPs exhibited more than three-fold stronger fluorescence intensity than free cypate at any given equal concentration. This is possibly because, like many other carbocyanine dyes such as ICG, free cypate, which is even more hydrophobic than ICG, might form self-quenched, weakly fluorescent $H$-aggregates in an aqueous environment.[42-44] In contrast, $\mathrm{SP}^{3} \mathrm{NPs}$ retained the strong fluorescence of pristine cypate under physiological conditions, suggesting that they are suitable for use as a NIR imaging probe.

A

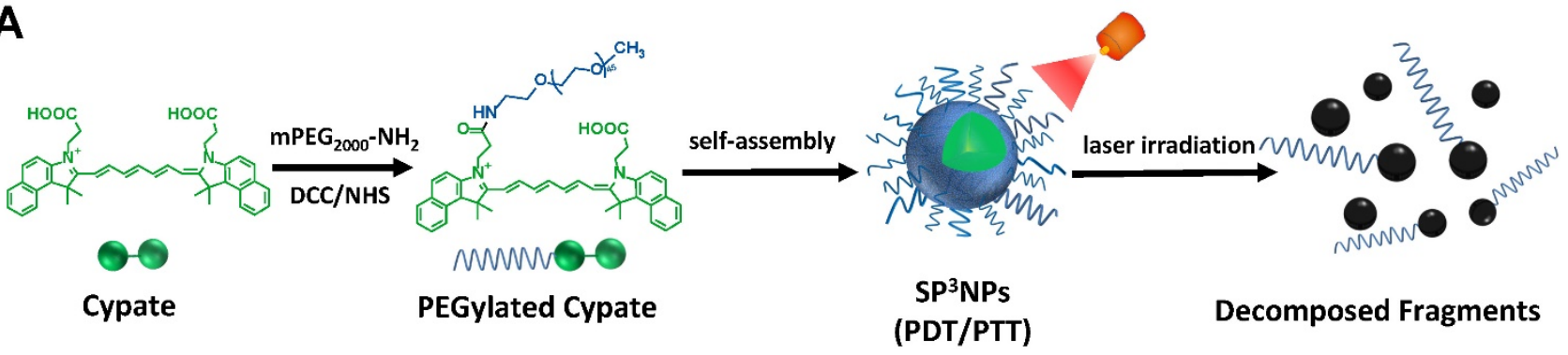

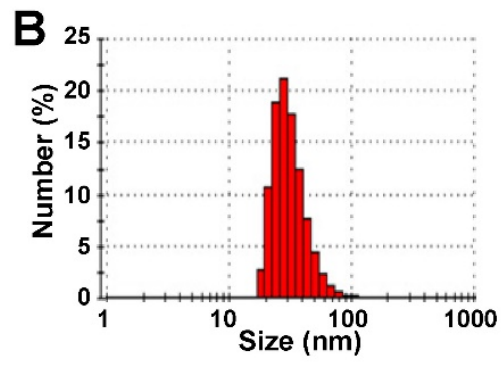

C
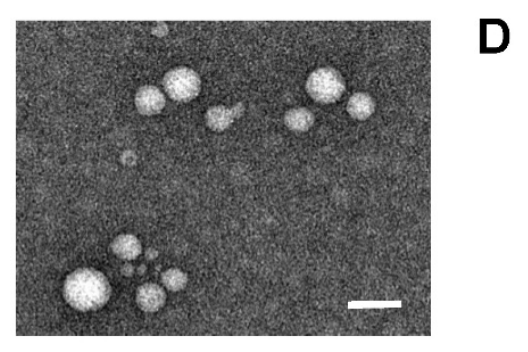

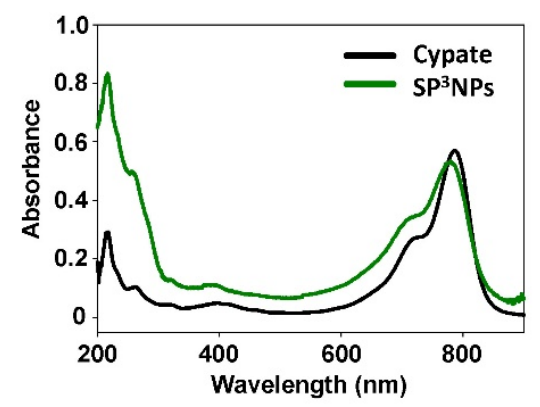

E

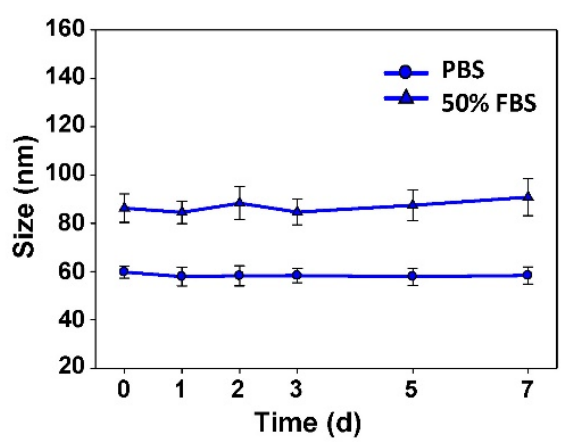

$\mathbf{F}$

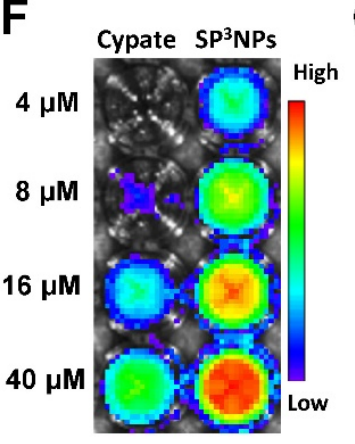

$\mathbf{G}$

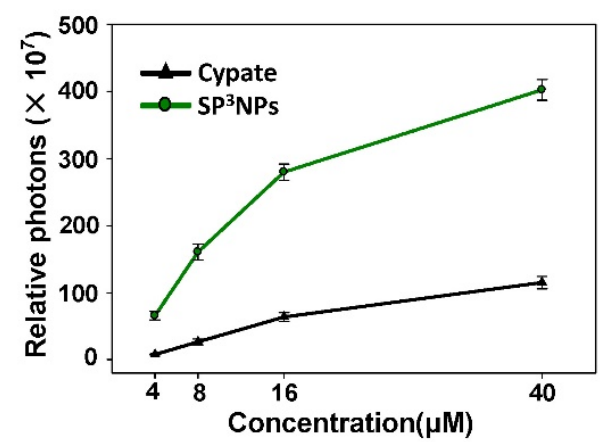

Figure 1. Schematic illustration and characterization of SP3NPs for tumor imaging and phototherapy. (A) Schematic illustration of synthesis of PEGylated cypate, its self-assembled nanoparticles, SP3NPs, and laser-triggered decomposition of the nanoparticles. (B) DLS data showing the hydrodynamic size of SP3NPs in PBS (pH 7.4). TEM image (C) and UV-Vis-NIR spectrum (D) of SP3NPs. Scale bar: $100 \mathrm{~nm}$. (E) Colloidal stability of SP3NPs in PBS and 50\% FBS, respectively. (F) Fluorescence images of cypate and serial dilutions of SP3NPs in PBS (Ex/Em: 710-760 nm/810-875 nm). (G)Semi-quantitation of fluorescence intensity in each region of interest (ROI) of Figure 1F. 

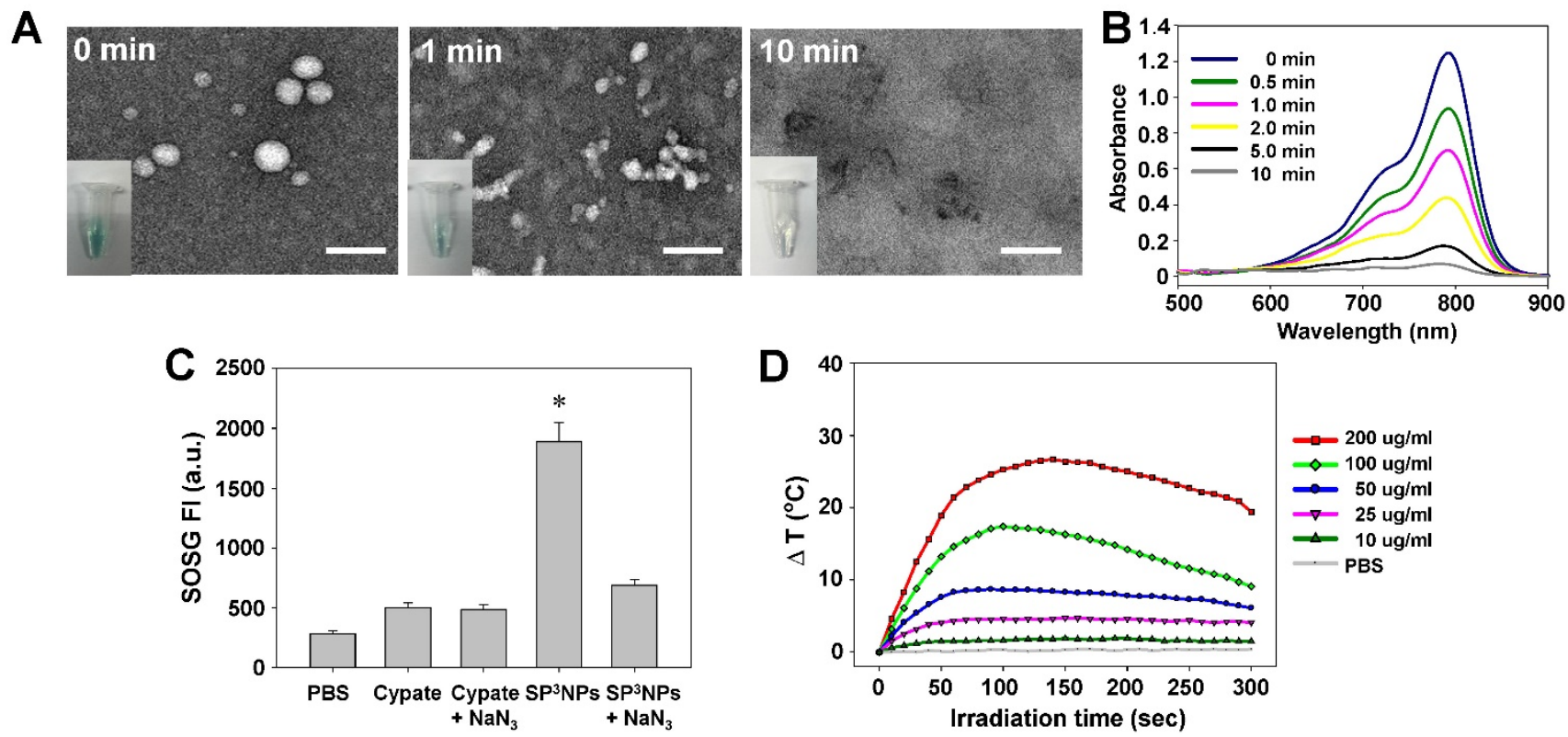

Figure 2. NIR irradiation-triggered decomposition and photo-properties of SP3NPs. (A) TEM images of SP3NPs upon NIR irradiation over time (808 $\mathrm{nm}$, $0.8 \mathrm{~W} \mathrm{~cm}^{-2}$ ). Insets show digital images of SP3NPs in PBS at the corresponding time points. Scale bar: $100 \mathrm{~nm}$. (B) Changes in UV-Vis spectra of SP3NPs upon NIR laser irradiation. (C) SOSG fluorescence intensity (FI) at $525 \mathrm{~nm}(\mathrm{Ex}: 488 \mathrm{~nm})$ of PBS, free cypate and SP3NPs, with or without sodium azide (100 $\mu M)$, after 808 -nm laser irradiation $\left(0.8 \mathrm{~W} \mathrm{~cm}^{-2}, 5 \mathrm{~min}\right)$. ${ }^{*} \mathrm{p}<0.05$ compared to other groups. (D) Photothermal heating curves of SP3NPs at different concentrations under $808-\mathrm{nm}$ laser irradiation for $5 \mathrm{~min}\left(0.8 \mathrm{~W} \mathrm{~cm}^{-2}\right)$.

\section{Photo-induced decomposition of SP ${ }^{3}$ NPs}

The possibility that $\mathrm{SP}^{3} \mathrm{NPs}$ undergo photo-induced decomposition like ICG was explored. As shown in Figure 2A, upon NIR irradiation with a continuous-wave laser $\left(808 \mathrm{~nm}, 0.8 \mathrm{~W} \mathrm{~cm}^{-2}\right)$, SP ${ }^{3} \mathrm{NPs}$ underwent significant, irreversible morphological changes, even following a 1-min exposure; at $10 \mathrm{~min}$ post-irradiation, no detectable nanoparticles remained. This NIR-induced photo-decomposition was accompanied by fast decoloration (Figure 2A, insets), with only $5.5 \%$ of the characteristic absorption peak for $\mathrm{SP}^{3} \mathrm{NPs}$ at $790 \mathrm{~nm}$ remaining at $10 \mathrm{~min}$ post-irradiation (Figure 2B). Such rapid photo-decomposition and photobleaching are presumably attributable to NIR-triggered destruction of the cypate chromophore structure,[30,44] leaving behind several fragments of PEGylated cypate with increased hydrophilicity, thereby resulting in disruption of SP3NPs. This NIR-induced decomposition and disruption behavior suggests that SP ${ }^{3}$ Ps could be easily excreted from the body after treatment with a NIR laser, thus alleviating toxicity concerns. With the rapid development and wider applications of nanomedicines, the biological effect and safety issue of them has drawn considerable attention recently. Even the in vivo fate of SP ${ }^{3} \mathrm{NPs}$ still required further study in detail, the strategy using NIR light as an external stimulus to control the metabolism and elimination behavior of nanomaterials could offer a new avenue for that concern. Moreover, compared to conventional UV light-responsive nanoparticles, our SP ${ }^{3} \mathrm{NPs}$ obviously have favorable properties for the imaging-based diagnosis and prognosis, especially for deep-sited diseases. Moreover, it is likely that SP3 NPs can be used as a photo-responsive spatiotemporal controlled drug-delivery carrier after loading therapeutic cargos.

\section{Generation of singlet oxygen and heat upon NIR irradiation}

Next we examined the ability of $\mathrm{SP}^{3} \mathrm{NPs}$ to generate both singlet oxygen $\left({ }^{1} \mathrm{O}_{2}\right)$ for a photodynamic effect and heat for photothermal therapy in response to NIR laser irradiation. As shown in Figure 2C, exposure to laser irradiation at $808 \mathrm{~nm}$ generated ${ }^{1} \mathrm{O}_{2}$, determined by measuring the fluorescence intensity of SOSG. SP ${ }^{3} \mathrm{NPs}$ generated much stronger $(\sim 3.8$-fold $)$ SOSG fluorescence intensity compared with free cypate. This is resulted from the hydrophobic free cypate easily forms H-aggregate, and strong intramolecular Förster resonance energy transfer (FRET) effect due to close proximity. Different from the physical entrapment of ICG insides of nanoparticles, which often leads to self-quenching,[11, 35, 37-38] the high fluorescence and ${ }^{1} \mathrm{O}_{2}$ generation of $\mathrm{SP}^{3} \mathrm{NPs}$ probably suggests that the cypate moieties in $\mathrm{SP}^{3} \mathrm{NPs}$ are not close packed. But the accurate nanostructure and assembly style of $\mathrm{SP}^{3} \mathrm{NPs}$ still needs further identification. This SOSG was significantly reduced in the presence of $\mathrm{NaN}_{3}$, a typical ${ }^{1} \mathrm{O}_{2}$ scavenger, supporting the conclusion that 
SP ${ }^{3} \mathrm{NPs}$ have the ability to produce ${ }^{1} \mathrm{O}_{2}$ in response to NIR laser irradiation. But the addition of $\mathrm{NaN}_{3}$ did not further reduce the SOSG of cypate, which was likely due to the background interference of ${ }^{1} \mathrm{O}_{2}$ indicator.

We next explored whether $\mathrm{SP}^{3} \mathrm{NPs}$ could also generate heat in response to NIR laser irradiation. The temperature of sample solutions during NIR laser irradiation at $808 \mathrm{~nm}$ was recorded as a function of $\mathrm{SP}^{3} \mathrm{NPs}$ concentration using a real-time IR thermal imaging system (Figure 2D). We found that, with increasing concentrations of $\mathrm{SP}^{3} \mathrm{NPs}$, the temperature rapidly rose and reached a plateau within 2 min of irradiation, producing $17.4{ }^{\circ} \mathrm{C}$ and $25.3{ }^{\circ} \mathrm{C}$ increases $(\Delta \mathrm{T})$ at SP${ }^{3} \mathrm{NPs}$ concentrations of 100 and $200 \mu \mathrm{g} \mathrm{ml}^{-1}$, respectively. The PBS control showed no such photothermal effect. These observations indicate that $\mathrm{SP}^{3} \mathrm{NPs}$ also possess sufficient enough photothermal property able to damage cancer cells. We speculate that like ICG, the excited energy state of cypate in $\mathrm{SP}^{3} \mathrm{NPs}$ could be partially transited to production of ${ }^{3} \mathrm{O}_{2}$ and at the same time partially converted into heat through vibronic relaxation or other non-irradiative transition pathway in parallel, leading to the dual PDT/PTT behavior.[45] However, like ICG, SP ${ }^{3}$ PPs also could be decomposed by light through self-sensitized photo oxidation process; in other words, the decomposition of SP3NPs could be catalyzed by the generated ${ }^{1} \mathrm{O}_{2}$ in the photodynamic type 2 reaction.[30] This NIR-induced photo-bleaching and photo-decomposition of SP3NPs resulted in gradual decrease in the heat (temperature) generated by $\mathrm{SP}^{3} \mathrm{NPs}$ after reaching a peak (Figure 2D).[46] Taken together, this NIR-induced simultaneous and efficient generation of both heat and ${ }^{1} \mathrm{O}_{2}$ suggests the potential use of $\mathrm{SP}^{3} \mathrm{NPs}$ in combined PDT and PTT for effective cancer therapy.

\section{Efficient cell entry of SP'NPs and NIR irradiation-triggered intracellular generation of ROS and heat}

The cellular uptake efficiency of $\mathrm{SP}^{3} \mathrm{NPs}$ in cancer cells was assessed in B16F10 murine melanoma cells using confocal laser scanning microscopy (CLSM) and flow cytometry. Near infrared red fluorescence corresponding to $\mathrm{SP}^{3} \mathrm{NPs}$ was observed in the cytosol of most cells treated with the nanoparticles, whereas little red fluorescence was seen in cells treated with free cypate (Figure 3A). A flow cytometry analysis further confirmed the significantly greater cellular uptake of $\mathrm{SP}^{3} \mathrm{NPs}$ by cancer cells compared with free cypate (Figure 3B). The limited cellular internalization of free cypate might be ascribed to its nonspecific binding to serum proteins, as is the case for other carbocyanine dyes. $[15,47]$

Next, the level of intracellular ROS generation in SP ${ }^{3}$ NPs-treated cancer cells upon exposure to a NIR laser at $0.8 \mathrm{~W} \mathrm{~cm}^{-2}$ for $3 \mathrm{~min}$ was assessed using the ROS-detecting fluorescent probe, 2',7'-dichlorodihydrofluorescein diacetate $\left(\mathrm{H}_{2} \mathrm{DCFDA}\right)$. As expected, $\mathrm{SP}^{3} \mathrm{NPs}$-treated cancer cells exhibited strong fluorescence as a result of activation of $\mathrm{H}_{2} \mathrm{DCFDA}$, indicative of a high level of intracellular ROS generation (Figure 3C). No ROS was generated in $\mathrm{SP}^{3} \mathrm{NPs}$-treated cells in the absence of NIR irradiation (Figure S2A). Furthermore, co-treatment with either glutathione or sodium azide $\left({ }^{1} \mathrm{O}_{2}\right.$ scavengers $)$ decreased the intracellular fluorescence intensity to background levels, whereas D-mannitol, a scavenger of hydroxyl radicals, did not affect fluorescence intensity, further confirming that ${ }^{1} \mathrm{O}_{2}$ is the main type of intracellular ROS produced by irradiation of $\mathrm{SP}^{3} \mathrm{NPs}$. This increase in ROS generation was accompanied by an increase in the temperature of $\mathrm{SP}^{3} \mathrm{NPs}$-treated cells to $41.2{ }^{\circ} \mathrm{C}$, an effect that was largely absent in free cypate-treated cells (Figure 3D, Figure S3). Since SP NPs could generate both ${ }^{1} \mathrm{O}_{2}$ and heat inside cells upon exposure to NIR, we evaluated their cancer cell-killing effect in B16F10 mouse melanoma cells as a function of $\mathrm{SP}^{3} \mathrm{NPs}$ concentration and laser intensity using MTT assays. For free cypate, its photo-induced anticancer effect is negligible, probably resulting from its poor cell entry and limited intracellular generation of ROS and heat (Figure 3E). However, as shown in Figure 3F, the cytotoxicity of $\mathrm{SP}^{3} \mathrm{NPs}$ was quite significant, and positively correlated with both the concentration of SP NPs and the laser power used. As expected, a higher concentration of the nanoparticles with higher light power resulted in a greater anticancer effect. However, SP ${ }^{3} \mathrm{NPs}$ caused little cytotoxicity in the absence of NIR irradiation. Whereas addition of hydroxyl radical scavengers (sodium pyruvate or D-mannitol) did not affect cell viability, co-treatment with ${ }^{1} \mathrm{O}_{2}$ scavengers (glutathione or sodium azide) significantly decreased $\mathrm{SP}^{3} \mathrm{NPs}$-induced cytotoxicity (Figure S2C). In addition, we confirmed that the fragmented, decomposed products of $\mathrm{SP}^{3} \mathrm{NPs}$ that appeared upon NIR irradiation were not cytotoxic (Figure S4), implying that, after exerting their NIR light-induced anticancer effects, $\mathrm{SP}^{3} \mathrm{NPs}$ degrade into less toxic fragments. These in vitro cell studies demonstrate that $\mathrm{SP}^{3} \mathrm{NPs}$ could be efficiently taken up by cancer cells and, following NIR irradiation, could kill cancer cells through the combined effects of intracellular generation of ${ }^{1} \mathrm{O}_{2}$ and hyperthermia. 
A
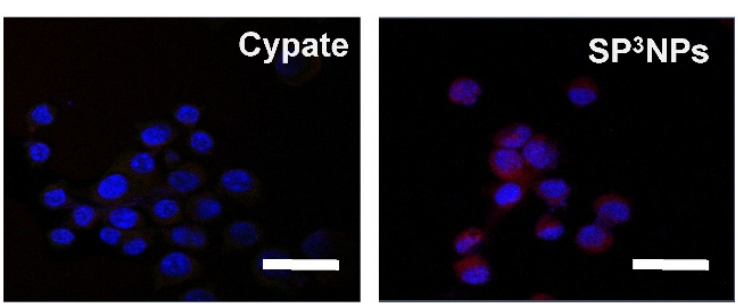

B

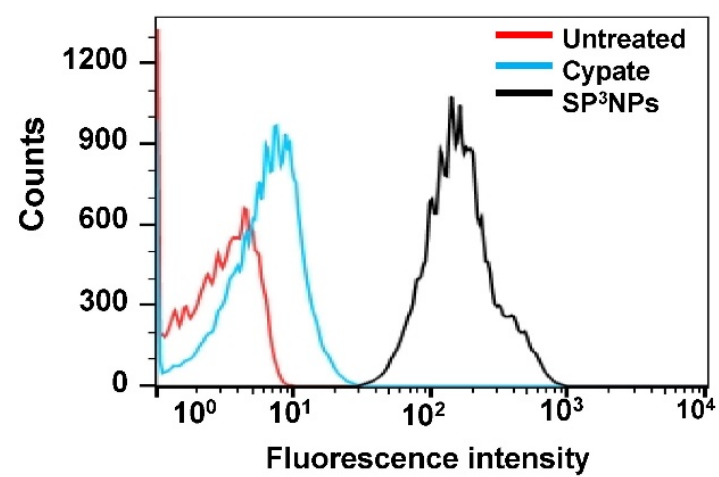

E

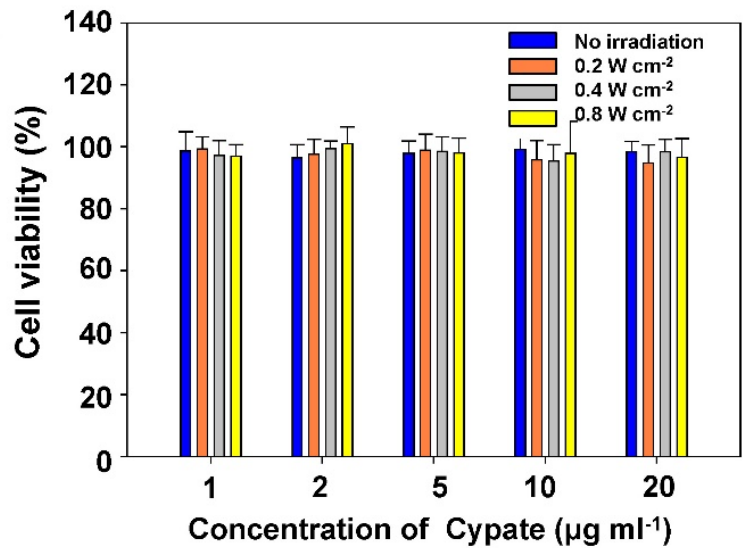

C
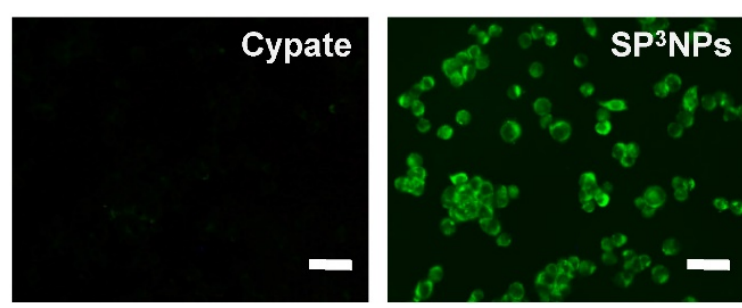

D
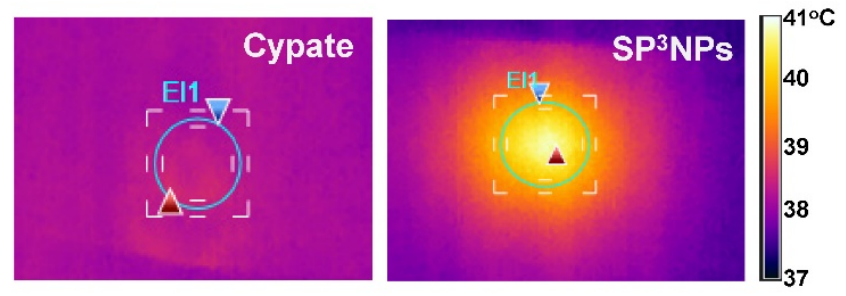

$F$

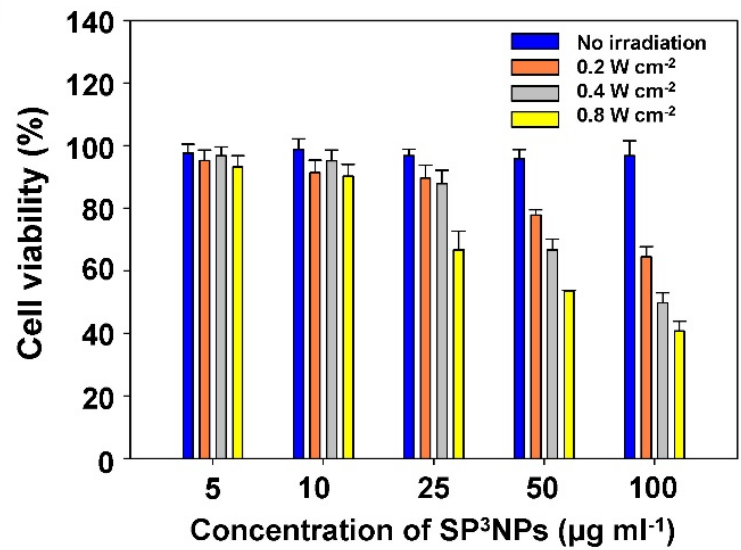

Figure 3. Cellular internalization, NIR light-induced ROS and heat generation, and cytotoxicity of SP3NPs in vitro. CLSM images (A) and flow cytometry analyses (B) of B16F10 melanoma cells treated with free cypate or SP3NPs for $1 \mathrm{~h}$. Cypate- or SP3NPs-treated cells were irradiated with an 808-nm laser $\left(0.8 \mathrm{~W} \mathrm{~cm}^{-2}, 3 \mathrm{~min}\right)$. Intracellular ROS generation $(C)$ was detected with $\mathrm{H}_{2}$ DCFDA $(10 \mu \mathrm{M})$. After irradiation, thermal images of cells (D) were recorded using an IR thermal camera. Relative viabilities of B16F10 cells treated with different concentrations of free cypate (E) and SP3NPs (F) for 1 h, followed by $808-\mathrm{nm}$ laser irradiation ( $3 \mathrm{~min}$ at $0.2,0.4$ or $0.8 \mathrm{~W} \mathrm{~cm}^{-2}$ ) and further incubation for $24 \mathrm{~h}$.

\section{Biodistribution and tumor localization of SP3NPs}

It was anticipated that SPNPs could preferentially localize at tumor sites through the EPR effect owing to their relatively small size $(\sim 60 \mathrm{~nm})$ and highly PEGylated surface. To test this, we administered free cypate or SP3NPs $\left(0.25 \mathrm{mg} \mathrm{kg}^{-1}\right.$ equivalent to cypate) via the tail vein to mice bearing B16F10 melanoma allografts. Figure 4A shows the real-time biodistribution and tumor accumulation at various time points. Only a weak fluorescence signal was observed in the tumor region in mice treated with free cypate. In contrast, SP ${ }^{3} \mathrm{NPs}$ exhibited much higher fluorescence signal in the tumor area as well as prolonged circulation/retention in the body over $24 \mathrm{~h}$ compared with free cypate. After $4 \mathrm{~h}$, a difference in tumor-to-background intensity became apparent, clearly delineating the tumor region. Time-course measurements of photon counts in tumor sites showed that, at $1 \mathrm{~h}$ post-injection, the fluorescence intensity of tumors in SPNPs-treated mice was $\sim 18$ times stronger than that in mice treated with free cypate (Figure 4B). The ex vivo biodistribution image (Figure S5) also revealed the greater tumor accumulation of $\mathrm{SP}^{3} \mathrm{NPs}$ than that of cypate. Furthermore, we observed the considerable distribution of SP3NPs in liver, which probably suggests $\mathrm{SP}^{3} \mathrm{NPs}$ are mainly excreted through liver. Tumor tissues were excised at $4 \mathrm{~h}$ post-injection and cryosectioned. CLSM imaging showed that $\mathrm{SP}^{3} \mathrm{NPs}$ were evenly distributed within the tumor tissue with a far greater intensity than free cypate, particularly in the center region of the tumor (Figure 4C). The high degree of tumor accumulation and distribution 
observed with NIR-emitting $\mathrm{SP}^{3} \mathrm{NPs}$ is highly advantageous for imaging-guided, effective phototherapy of cancers.

\section{In vivo tumor ablation by multiple phototherapies of SP ${ }^{3}$ NPs}

Encouraged by tumor targeting and imaging results, we next examined the therapeutic efficacy of $\mathrm{SP}^{3} \mathrm{NPs}$ in combination with NIR laser irradiation in tumor-bearing mice. B16F10 melanoma-bearing mice (average tumor volume, $\sim 100 \mathrm{~mm}^{3}$ ) were randomly divided into six groups ( $n=6 /$ group): PBS, PBS + NIR, free cypate, free cypate + NIR, SP3NPs, and $\mathrm{SP}^{3} \mathrm{NPs}+\mathrm{NIR}$. SP ${ }^{3} \mathrm{NPs}$ or free cypate was administered as a single intravenous injection via the tail vein at a dose of $5 \mathrm{mg} \mathrm{kg}^{-1}$ equivalent of cypate, and tumor regions were locally irradiated with a NIR laser $\left(808 \mathrm{~nm}, 0.4 \mathrm{~W} \mathrm{~cm}^{-2}\right)$ for $10 \mathrm{~min}$ at $4 \mathrm{~h}$ post injection because the tumor-to-background intensity became apparent, clearly delineating the tumor region at the time point. The temperature at the tumor region was continuously monitored throughout NIR exposure with an IR thermal camera (Figure 5A). Mice treated with PBS showed negligible temperature increases in the tumor over the course of irradiation; however, mice in the SP3NPs-injected group exhibited significant increases in temperature, reaching $45^{\circ} \mathrm{C}$, a temperature much higher than that in mice injected with free cypate $\left(\sim 38^{\circ} \mathrm{C}\right)$ under the same treatment conditions (Figure 5B). Considering the fast oxygen depletion and severe hypoxia microenvironment occurs in tumor tissue - which could lead to unsatisfactory outcome of other therapies - when PDT is applied, [48] we regard PTT might play a more important role and result in better therapeutic outcome in vivo, especially for hypoxic tumor treatment.
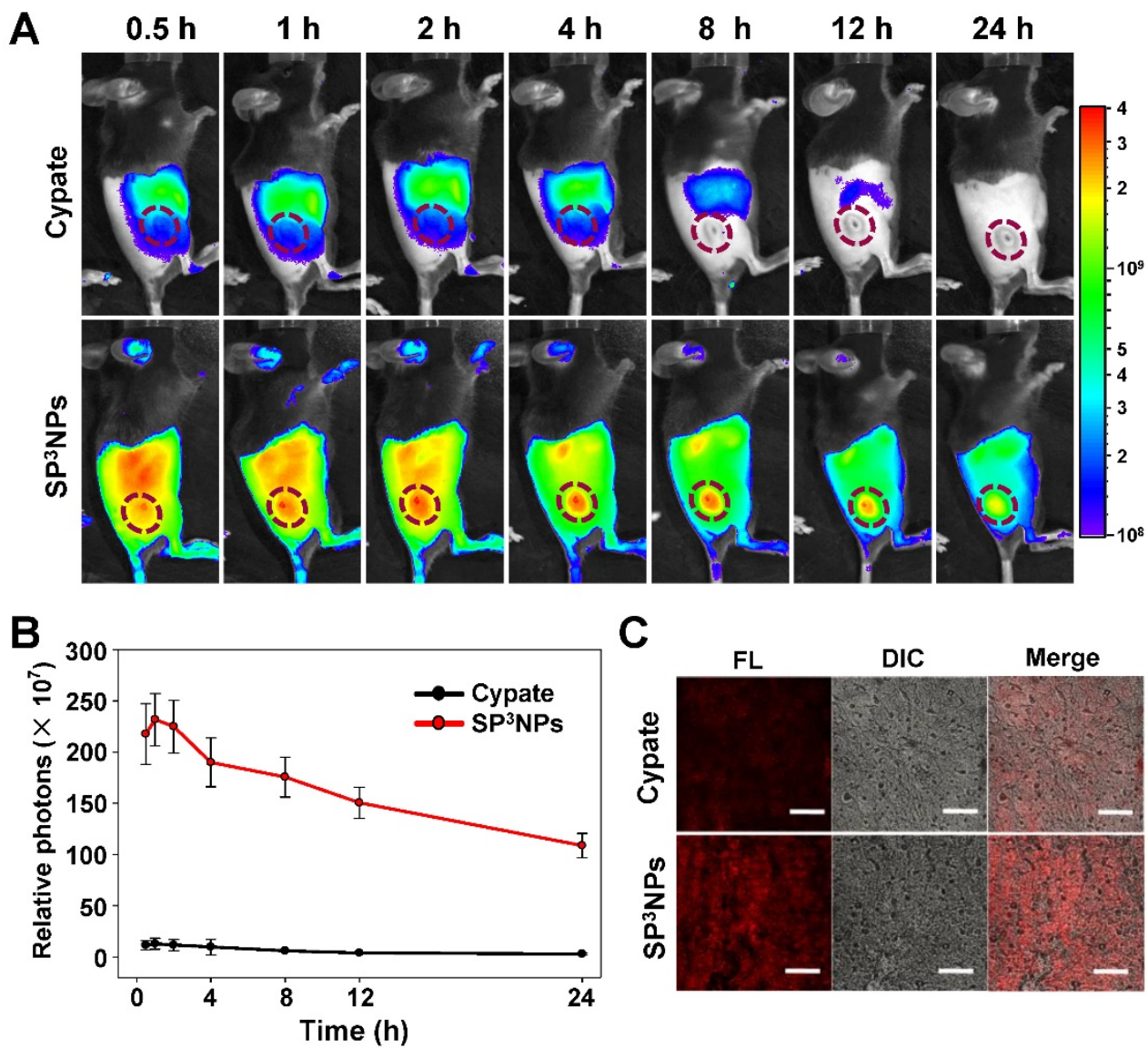

Figure 4. In vivo biodistribution of SP3NPs in tumor-bearing mice. (A) Whole-body NIR fluorescence images of B16F10 melanoma-bearing mice injected with free cypate or SPNNPs at a dose of $0.25 \mathrm{mg} \mathrm{kg}^{-1}$ cypate at $0.5,1,2,4,8,12$, and $24 \mathrm{~h}$ after injection via tail vein, respectively. (B) Semi-quantitative analysis of fluorescence intensity in tumor sites using Living Image Software Version $2.6(n=4)$. (C) CLSM images of cryosectioned tumor tissues, excised 4 h after intravenous administration of free cypate or SP ${ }^{3}$ NPs. Scale bar: $50 \mu \mathrm{m}$. 

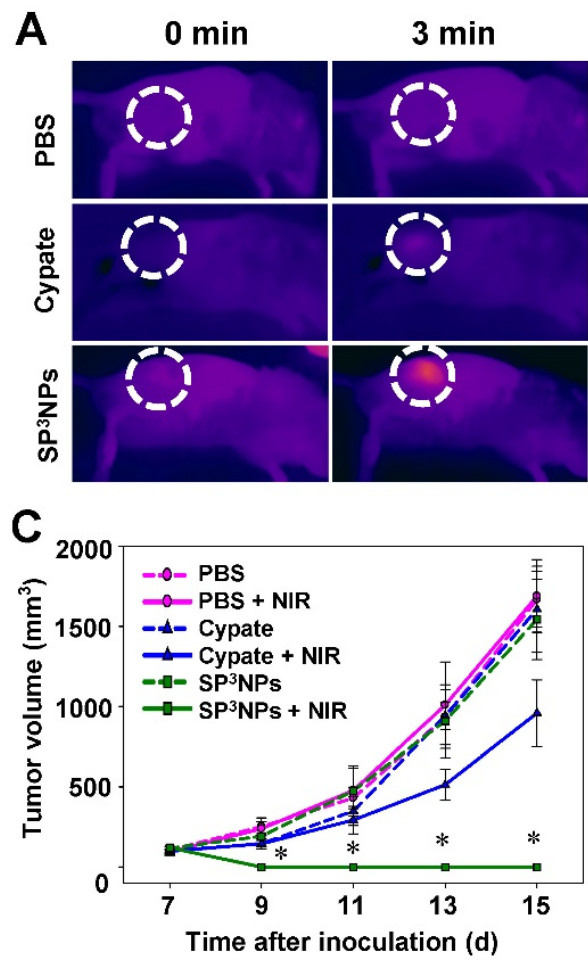

$3 \mathrm{~min}$
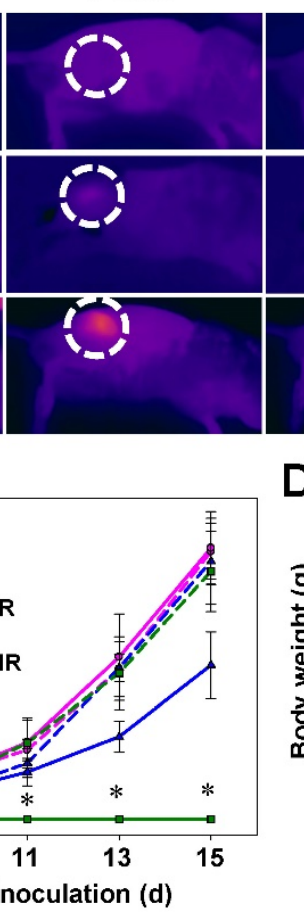

$6 \min$

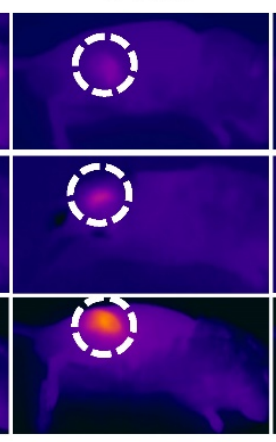

$D_{35}$

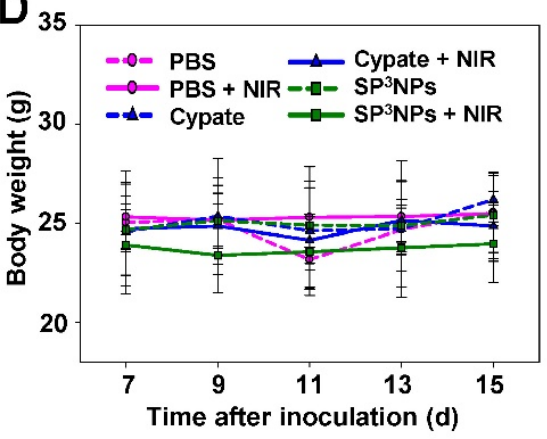

B
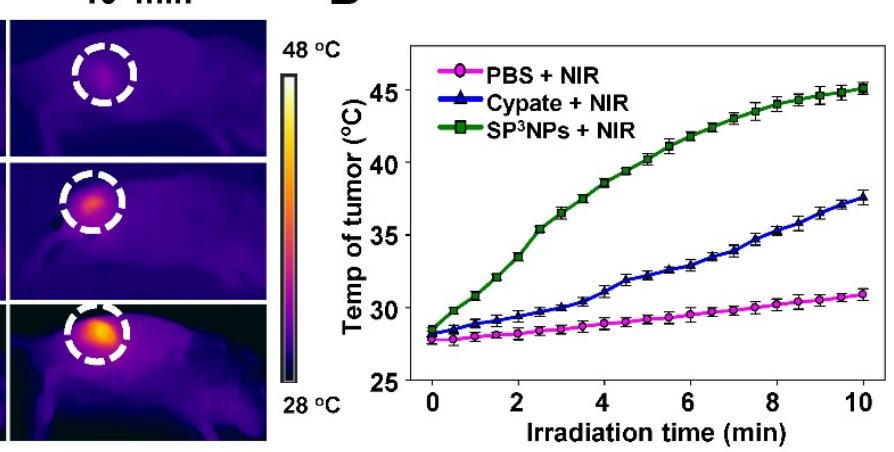

$E$

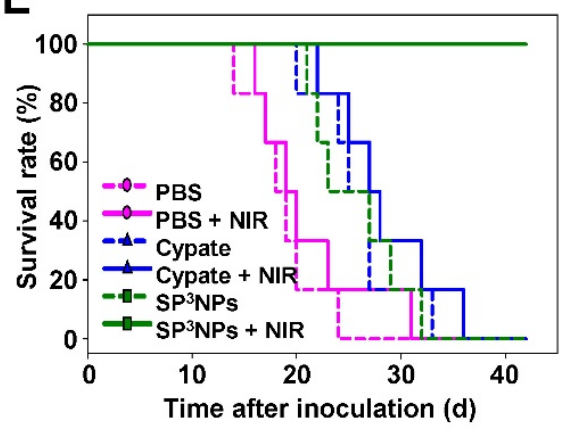

Figure 5. NIR laser-induced antitumor effects of SP3NPs. (A) Real-time thermal images of B16F10 melanoma-bearing mice exposed to 808 -nm laser (0.4 W $\mathrm{cm}^{-2}$ ) for $10 \mathrm{~min}$ at $4 \mathrm{~h}$ post-injection of PBS, free cypate, or SPN NPs ( $5 \mathrm{mg} \mathrm{kg}^{-1}$ cypate). The dashed circles indicate the locations of tumors. (B) Photothermal heating curves of tumors upon 808-nm laser irradiation for $10 \mathrm{~min}(n=3)$. Tumor growth curves $(C)$, body weight changes $(D)$ and survival rates $(E)$ of $B 16 \mathrm{~F} 10$ melanoma-bearing mice intravenously treated with PBS, free cypate or SPNNs (5 mg kg-1 cypate, single injection, $\mathrm{n}=6-7)$, with or without $808-\mathrm{nm}$ laser irradiation $\left(0.4 \mathrm{~W} \mathrm{~cm}^{-2}, 10 \mathrm{~min}\right) .{ }^{*} \mathrm{p}<0.05$ compared to other groups at a given time point.

Therapeutic efficacy following the single intravenous injection and subsequent NIR irradiation was evaluated by measuring tumor volume, body weight, and survival rate (Figure 5C, 5D, 5E and Figure S6). No inhibition of tumor growth was observed in the PBS alone or PBS + NIR groups, and tumor volumes in the two groups were similar. Tumor growth in both SPNNPs and free cypate-alone groups was also similar to that in the PBS control group, suggesting that neither treatment is cytotoxic in the absence of NIR light. In contrast, tumors in the $\mathrm{SP}^{3} \mathrm{NPs}+\mathrm{NIR}$ group were completely ablated, as determined by measurement of tumor volume as well as histological analyses (Figure 5C, and Figure 6). Furthermore, continuous monitoring revealed that no tumors recurred in this group up to $42 \mathrm{~d}$ later, resulting in a survival rate of $100 \%$ (Figure 5E). Mice in the SP NPs + NIR group also showed no signs of significant body weight loss, suggesting no acute side effects of the nanoparticles per se or the phototherapy (Figure 5D). Although treatment of mice with free cypate + NIR inhibited tumor growth by $\sim 57 \%$ relative to PBS controls, all mice in this group died due to tumor burden within $36 \mathrm{~d}$ (Figure 5E). Antitumor efficacy was further assessed by hematoxylin and eosin staining $(\mathrm{H} \& \mathrm{E})$ and terminal deoxynucleotidyl transferase dUTP nick-end labeling
(TUNEL) assays. As shown in Figure 6A and 6B, the tumor tissues of mice in PBS + NIR, free cypate and $\mathrm{SP}^{3} \mathrm{NPs}$-alone groups exhibited infiltration of cancer cells with highly pleomorphic nuclei and showed little evidence of apoptosis, results similar to those observed in the PBS control group. Only a few sporadic necrotic cells were found in tumor tissues from the free cypate + NIR-treated group, suggesting limited therapeutic efficacy. In contrast, tumor tissue sections from mice treated with $\mathrm{SP}^{3} \mathrm{NPs}+\mathrm{NIR}$ showed massive collapse of cell nuclei and considerable fragmented DNA in nucleus, indicating the death of tumor cells is likely through apoptosis way, as well as the potency of multiple phototherapies. It is also worth noting that the laser power intensity used in this study $\left(0.4 \mathrm{~W} \mathrm{~cm}^{-2}\right)$ is among the lowest reported $\left(\sim 1-2 \mathrm{~W} \mathrm{~cm}^{-2}\right)$ for in vivo PTT.[21,49-51] These results clearly suggest that $\mathrm{SP}^{3} \mathrm{NPs}$ can be preferentially delivered to the site of a tumor owing to the highly PEGylated nature of its surface and proper size, and following optical imaging-guided NIR laser irradiation, can lead to effective tumor ablation and prolonged survival of mice without tumor recurrence as a consequence of the synergistic antitumor efficacy of multiple phototherapies. 

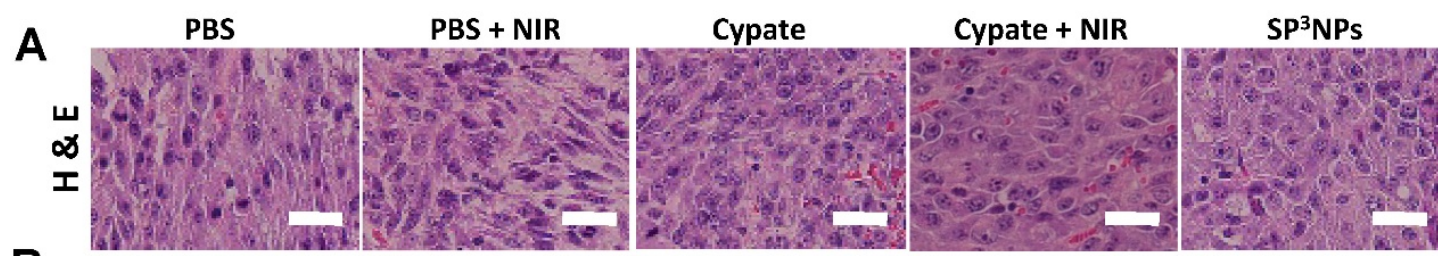

SP ${ }^{3} \mathrm{NPS}+\mathrm{NIR}$
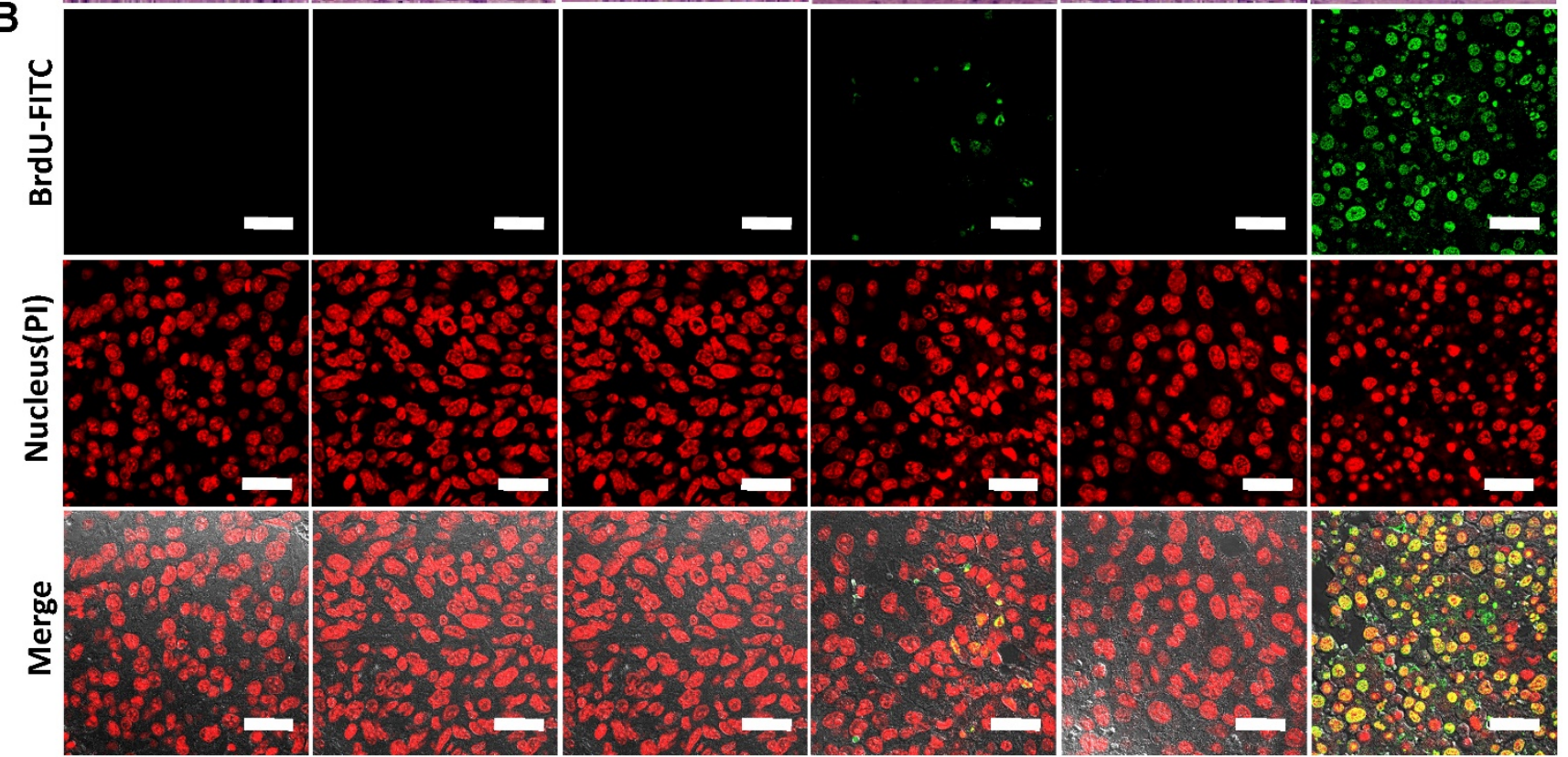

Figure 6. The multiple NIR-induced therapeutic effect of SP3NPs in tumor tissues. Tumor tissues were excised and sectioned for H\&E (A) and TUNEL (B) staining at $12 \mathrm{~h}$ post-irradiation. Scale bar: $25 \mu \mathrm{m}$.

\section{Conclusion}

In summary, we have successfully designed and fabricated a novel theranostic nanomedicine platform, $\mathrm{SP}^{3} \mathrm{NPs}$, with integrative cancer imaging and multiple phototherapy capabilities. Our experimental results clearly demonstrate that SPNPs strongly accumulated in tumor tissue owing to their highly PEGylated surface and relatively small size $(\sim 60 \mathrm{~nm})$, offering subsequent imaging-guided multiple phototherapies that resulted in tumor eradication and prolonged survival of mice. As a potential nanomedicine, $\mathrm{SP}^{3} \mathrm{NPs}$ possess several attributes that tend to favor translation to the clinic in the future. First, SP ${ }^{3}$ NPs can be easily prepared by self-assembly of solely PEGylated cypate of a single chemical entity, comprising FDA-approved PEG and an ICG derivative. Second, SPNNS exhibit intense NIR fluorescence following irradiation with much longer wavelengths $(\sim 800 \mathrm{~nm})$ than conventional dyes used for PDT in the clinic, suggesting the possibility of deeper tissue penetration. Lastly, the unique ability of SP NPs to undergo irreversible decomposition upon NIR laser irradiation may alleviate potential toxicity concerns associated with phototherapy. Taken together, our findings demonstrate that the simple and decomposable nanoparticles described here, which enable imaging-guided, multiple phototherapy, represent a novel and facile approach for next-generation theranostics with great promise for translation into clinical practice in the future.

\section{Supplementary Material}

Characterization of PEGylated cypate (Figure S1); Effect of various ROS scavengers on intracellularly generated ROS by $\mathrm{SP}^{3} \mathrm{NPs}$ upon NIR laser irradiation (Figure S2); Temperature change of cells treated with cypate and SP NPs during NIR irradiation (Figure S3); Assessment of cytotoxicity of NIR-decomposed fragments of SP3NPs (Figure S4); Ev vivo fluorescence image of five vital organs excising from cypate- and $\mathrm{SP}^{3} \mathrm{NPs}$-treated tumor-bearing mice (Figure S5); Digital photos of tumor-bearing mice after treatment with each regimen and NIR laser (Figure S6). http://www.thno.org/v06p2367s1.pdf

\section{Abbreviations}

PDT: photodynamic therapy; PTT: photothermal therapy; NIR light: near infrared light; ROS: reactive oxygen species; PS: photosensitizers; ${ }^{1} \mathrm{O}_{2}$ : singlet oxygen; ICG: indocyanine green; DCC: $\mathrm{N}, \mathrm{N}^{\prime}$-dicyclohexylcarbodiimide; NHS: N-hydroxysuccinimide; MTT: 3-(4,5-dime-thylthiazol-2-yl)-2,5diphenyltetrazolium bromide; DAPI: 4',6-diamidino- 
2-phenylindole dihydrochloride; SOSG: singlet Oxygen Sensor Green; $\mathrm{H}_{2}$ DCFDA: 2',7'-Dichlorodihydrofluorescein diacetate; FBS: fetal bovine serum; NMR: nuclear magnetic resonance; MALDI-TOF: matrix-assisted laser desorption/ionization-time of flight spectrometry; TEM: transmission electron microscopy; PBS: phosphate buffered saline; CLSM: confocal laser scanning microscopy; TUNEL: terminal deoxynucleotidyl transferase-mediated dUTP nick-end labeling.

\section{Acknowledgement}

This work was supported by Global Research Laboratory (grant no. 2014044002) through the National Research Foundation of Korea (NRF) funded by the Ministry of Science, ICT \& Future Planning and the Korea Health Technology R\&D Project (grant no. HI13C21810301) through the Korea Health Industry Development Institute (HHIDI) funded by the Ministry of Health \& Welfare, Republic of Korea.

\section{Competing Interest}

The authors have declared that no competing interests exist.

\section{References}

1. Kotagiri N, Sudlow GP, Akers WJ, Achilefu S. Breaking the depth dependency of phototherapy with Cerenkov radiation and low-radiance-responsive nanophotosensitizers. Nat Nano. 2015; 10:370-9.

2. Carter KA, Shao S, Hoopes MI, Luo D, Ahsan B, Grigoryants VM, et al. Porphyrin-phospholipid liposomes permeabilized by near-infrared light. Nat Commun. 2014; 5.

3. Song J, Yang X, Jacobson O, Huang P, Sun X, Lin L, et al. Ultrasmall Gold Nanorod Vesicles with Enhanced Tumor Accumulation and Fast Excretion from the Body for Cancer Therapy. Advanced Materials. 2015; 27: 4910-7.

4. Huang J, Guo M, Ke H, Zong C, Ren B, Liu G, et al. Rational Design and Synthesis of $\gamma \mathrm{Fe} 2 \mathrm{O} 3 @$ Au Magnetic Gold Nanoflowers for Efficient Cancer Theranostics. Advanced Materials. 2015; 27: 5049-56.

5. Wang Y, Yang T, Ke H, Zhu A, Wang Y, Wang J, et al. Smart Albumin-Biomineralized Nanocomposites for Multimodal Imaging and Photothermal Tumor Ablation. Advanced Materials. 2015; 27: 3874-82.

6. Deng H, Dai F, Ma G, Zhang X. Theranostic Gold Nanomicelles made from Biocompatible Comb-like Polymers for Thermochemotherapy and Multifunctional Imaging with Rapid Clearance. Advanced Materials. 2015; 27: 3645-53.

7. Coutier S, Mitra S, Bezdetnaya LN, Parache RM, Georgakoudi I, Foster TH, et al. Effects of Fluence Rate on Cell Survival and Photobleaching in Meta-Tetra-(hydroxyphenyl)chlorin-photosensitized Colo 26 Multicell Tumor Spheroids $\uparrow$. Photochemistry and Photobiology. 2001; 73: 297-303.

8. Sitnik TM, Hampton JA, Henderson BW. Reduction of tumour oxygenation during and after photodynamic therapy in vivo: effects of fluence rate. British Journal of Cancer. 1998; 77: 1386-94.

9. Fingar VH, Wieman TJ, Wiehle SA, Cerrito PB. The Role of Microvascular Damage in Photodynamic Therapy: The Effect of Treatment on Vessel Constriction, Permeability, and Leukocyte Adhesion. Cancer Research. 1992; 52: 4914-21.

10. Dolmans DEJGJ, Fukumura D, Jain RK. Photodynamic therapy for cancer. Nat Rev Cancer. 2003; 3: 380-7.

11. Zheng M, Yue C, Ma Y, Gong P, Zhao P, Zheng C, et al. Single-Step Assembly of DOX/ICG Loaded Lipid-Polymer Nanoparticles for Highly Effective Chemo-photothermal Combination Therapy. ACS Nano. 2013; 7: 2056-67.

12. Zhang Z, Wang L, Wang J, Jiang X, Li X, Hu Z, et al. Mesoporous Silica-Coated Gold Nanorods as a Light-Mediated Multifunctional Theranostic Platform for Cancer Treatment. Advanced Materials. 2012; 24: 1418-23.

13. Zhu A, Miao K, Deng Y, Ke H, He H, Yang T, et al. Dually $\mathrm{pH} /$ Reduction-Responsive Vesicles for Ultrahigh-Contrast Fluorescence Imaging and Thermo-Chemotherapy-Synergized Tumor Ablation. ACS Nano. 2015; 9: 7874-85.
14. Zhang Z, Wang J, Chen C. Near-Infrared Light-Mediated Nanoplatforms for Cancer Thermo-Chemotherapy and Optical Imaging. Advanced Materials. 2013; 25: 3869-80.

15. Jang B, Park J-Y, Tung C-H, Kim I-H, Choi Y. Gold Nanorod-Photosensitizer Complex for Near-Infrared Fluorescence Imaging and Photodynamic/Photothermal Therapy In Vivo. ACS Nano. 2011; 5: 1086-94.

16. Lv R, Yang P, He F, Gai S, Yang G, Dai Y, et al. An imaging-guided platform for synergistic photodynamic/photothermal/chemo-therapy with $\mathrm{pH} /$ temperature-responsive drug release. Biomaterials. 2015; 63: 115-27.

17. Lv R, Yang P, He F, Gai S, Li C, Dai Y, et al. A Yolk-like Multifunctional Platform for Multimodal Imaging and Synergistic Therapy Triggered by a Single Near-Infrared Light. ACS Nano. 2015; 9: 1630-47.

18. Kim Y-K, Na H-K, Kim S, Jang H, Chang S-J, Min D-H. One-Pot Synthesis of Multifunctional Au@Graphene Oxide Nanocolloid Core@Shell Nanoparticles for Raman Bioimaging, Photothermal, and Photodynamic Therapy. Small. 2015; 11: 2527-35.

19. Song X, Liang C, Gong H, Chen Q, Wang C, Liu Z. Photosensitizer-Conjugated Albumin-Polypyrrole Nanoparticles for Imaging-Guided In Vivo Photodynamic/Photothermal Therapy. Small. 2015; 11: 3932-41.

20. Vijayaraghavan P, Liu C-H, Vankayala R, Chiang C-S, Hwang KC. Designing Multi-Branched Gold Nanoechinus for NIR Light Activated Dual Modal Photodynamic and Photothermal Therapy in the Second Biological Window. Advanced Materials. 2014; 26: 6689-95.

21. Wang S, Huang P, Nie L, Xing R, Liu D, Wang Z, et al. Single Continuous Wave Laser Induced Photodynamic/Plasmonic Photothermal Therapy Using Photosensitizer-Functionalized Gold Nanostars. Advanced Materials. 2013; 25: 3055-61.

22. Wang S, Riedinger A, Li H, Fu C, Liu H, Li L, et al. Plasmonic Copper Sulfide Nanocrystals Exhibiting Near-Infrared Photothermal and Photodynamic Therapeutic Effects. ACS Nano. 2015; 9: 1788-800.

23. Deng K, Hou Z, Deng X, Yang P, Li C, Lin J. Enhanced Antitumor Efficacy by $808 \mathrm{~nm}$ Laser-Induced Synergistic Photothermal and Photodynamic Therapy Based on a Indocyanine-Green-Attached $\mathrm{W}_{18} \mathrm{O}_{49}$ Nanostructure. Advanced Functional Materials. 2015; 25: 7280-90.

24. Tian B, Wang C, Zhang S, Feng L, Liu Z. Photothermally Enhanced Photodynamic Therapy Delivered by Nano-Graphene Oxide. ACS Nano. 2011; 5: 7000-9.

25. Gollavelli G, Ling Y-C. Magnetic and fluorescent graphene for dual modal imaging and single light induced photothermal and photodynamic therapy of cancer cells. Biomaterials. 2014; 35: 4499-507.

26. Wang Y, Wang H, Liu D, Song S, Wang X, Zhang H. Graphene oxide covalently grafted upconversion nanoparticles for combined NIR mediated imaging and photothermal/photodynamic cancer therapy. Biomaterials. 2013; 34: 7715-24.

27. Bäumler W, Abels $C$, Karrer $S$, Weiß $T$, Messmann $H$, Landthaler $M$, et al. Photo-oxidative killing of human colonic cancer cells using indocyanine green and infrared light. British Journal of Cancer. 1999; 80: 360-3.

28. Bozkulak O, Yamaci RF, Tabakoglu O, Gulsoy M. Photo-toxic effects of 809-nm diode laser and indocyanine green on MDA-MB231 breast cancer cells. Photodiagnosis and Photodynamic Therapy. 2009; 6: 117-21.

29. Schaafsma BE, Mieog JSD, Hutteman M, van der Vorst JR, Kuppen PJK, Löwik CWGM, et al. The clinical use of indocyanine green as a near-infrared fluorescent contrast agent for image-guided oncologic surgery. Journal of Surgical Oncology. 2011; 104: 323-32.

30. Engel E, Schraml R, Maisch T, Kobuch K, König B, Szeimies R-M, et al. Light-induced decomposition of indocyanine green. Investigative ophthalmology \& visual science. 2008; 49: 1777-83.

31. Ma Y, Tong S, Bao G, Gao C, Dai Z. Indocyanine green loaded SPIO nanoparticles with phospholipid-PEG coating for dual-modal imaging and photothermal therapy. Biomaterials. 2013; 34: 7706-14.

32. Kuo W-S, Chang Y-T, Cho K-C, Chiu K-C, Lien C-H, Yeh C-S, et al. Gold nanomaterials conjugated with indocyanine green for dual-modality photodynamic and photothermal therapy. Biomaterials. 2012; 33: 3270-8.

33. Sheng Z, Hu D, Zheng M, Zhao P, Liu H, Gao D, et al. Smart Human Serum Albumin-Indocyanine Green Nanoparticles Generated by Programmed Assembly for Dual-Modal Imaging-Guided Cancer Synergistic Phototherapy. ACS Nano. 2014; 8: 12310-22.

34. Miao W, Shim G, Kim G, Lee S, Lee H-J, Kim YB, et al. Image-guided synergistic photothermal therapy using photoresponsive imaging agent-loaded graphene-based nanosheets. Journal of Controlled Release. 2015; 211: 28-36.

35. Yang $\mathrm{H}$, Mao $\mathrm{H}$, Wan $\mathrm{Z}$, Zhu A, Guo M, Li Y, et al. Micelles assembled with carbocyanine dyes for theranostic near-infrared fluorescent cancer imaging and photothermal therapy. Biomaterials. 2013; 34: 9124-33.

36. Li Y, Deng Y, Tian X, Ke H, Guo M, Zhu A, et al. Multipronged Design of Light-Triggered Nanoparticles To Overcome Cisplatin Resistance for Efficient Ablation of Resistant Tumor. ACS Nano. 2015; 9: 9626-37.

37. Yan L, Qiu L. Indocyanine green targeted micelles with improved stability for near-infrared image-guided photothermal tumor therapy. Nanomedicine. 2015; 10: 361-73

38. Zhao P, Zheng M, Yue C, Luo Z, Gong P, Gao G, et al. Improving drug accumulation and photothermal efficacy in tumor depending on size of ICG loaded lipid-polymer nanoparticles. Biomaterials. 2014; 35: 6037-46. 
39. Huang P, Rong P, Jin A, Yan X, Zhang MG, Lin J, et al. Dye-Loaded Ferritin Nanocages for Multimodal Imaging and Photothermal Therapy. Advanced Materials. 2014; 26: 6401-8.

40. Ye Y, Bloch S, Kao J, Achilefu S. Multivalent Carbocyanine Molecular Probes: Synthesis and Applications. Bioconjugate Chemistry. 2005; 16: 51-61.

41. Miao W, Shim G, Lee S, Lee S, Choe YS, Oh Y-K. Safety and tumor tissue accumulation of pegylated graphene oxide nanosheets for co-delivery of anticancer drug and photosensitizer. Biomaterials. 2013; 34: 3402-10.

42. Desmettre T, Devoisselle JM, Mordon S. Fluorescence Properties and Metabolic Features of Indocyanine Green (ICG) as Related to Angiography. Survey of Ophthalmology. 2000; 45: 15-27.

43. Saxena V, Sadoqi M, Shao J. Degradation kinetics of indocyanine green in aqueous solution. Journal of Pharmaceutical Sciences. 2003; 92: 2090-7.

44. Philip R, Penzkofer A, Bäumler W, Szeimies RM, Abels C. Absorption and fluorescence spectroscopic investigation of indocyanine green. Journal of Photochemistry and Photobiology A: Chemistry. 1996; 96: 137-48.

45. Yuan A, Wu J, Tang X, Zhao L, Xu F, Hu Y. Application of near-infrared dyes for tumor imaging, photothermal, and photodynamic therapies. Journal of Pharmaceutical Sciences. 2013; 102: 6-28.

46. Zheng X, Zhou F, Wu B, Chen WR, Xing D. Enhanced Tumor Treatment Using Biofunctional Indocyanine Green-Containing Nanostructure by Intratumoral or Intravenous Injection. Molecular Pharmaceutics. 2012; 9: 514-22.

47. Berezin MY, Guo K, Akers W, Livingston J, Solomon M, Lee H, et al. Rational Approach To Select Small Peptide Molecular Probes Labeled with Fluorescent Cyanine Dyes for in Vivo Optical Imaging. Biochemistry. 2011; 50: 2691-700.

48. Jin CS, Lovell JF, Chen J, Zheng G. Ablation of Hypoxic Tumors with Dose-Equivalent Photothermal, but Not Photodynamic, Therapy Using a Nanostructured Porphyrin Assembly. ACS Nano. 2013; 7: 2541-50.

49. Yuan A, Qiu X, Tang X, Liu W, Wu J, Hu Y. Self-assembled PEG-IR-780-C13 micelle as a targeting, safe and highly-effective photothermal agent for in vivo imaging and cancer therapy. Biomaterials. 2015; 51: 184-93.

50. Yang K, Zhang S, Zhang G, Sun X, Lee S-T, Liu Z. Graphene in Mice: Ultrahigh In Vivo Tumor Uptake and Efficient Photothermal Therapy. Nano Letters. 2010; 10: 3318-23.

51. von Maltzahn G, Park J-H, Agrawal A, Bandaru NK, Das SK, Sailor MJ, et al. Computationally Guided Photothermal Tumor Therapy Using Long-Circulating Gold Nanorod Antennas. Cancer Research. 2009; 69: 3892-900. 